\title{
Nutritional Situation of Rural Communities in Nariño, Colombia 2013
}

\author{
Sara Eloísa Del Castillo Matamoros ${ }^{1,2}$, Teresa Mosquera Vásquez ${ }^{3}$, \\ Eliana Lorena Suárez Higuera ${ }^{3}$, Ana Patricia Heredia Vargas ${ }^{3}$ \\ ${ }^{1}$ Department of Human Nutrition, National University of Colombia, Bogotá, Colombia \\ ${ }^{2}$ Food and Nutrition Security Observatory (OBSAN), National University of Colombia, Bogotá, Colombia \\ ${ }^{3}$ Faculty of Agricultural Sciences, National University of Colombia, Bogotá, Colombia \\ Email: sedelcastillom@unal.edu.co, tmosquerav@unal.edu.co, elsuarezh@gmail.com, \\ patricia.heredia@gmail.com
}

Received 30 May 2014; revised 5 July 2014; accepted 18 July 2014

Copyright (C) 2014 by authors and Scientific Research Publishing Inc.

This work is licensed under the Creative Commons Attribution International License (CC BY). http://creativecommons.org/licenses/by/4.0/

(c) (i) Open Access

\section{Abstract}

Objective: To evaluate the nutritional situation by anthropometry of the rural families population aged 0 to 64 years in the municipalities of Pasto, Túquerres, Guachucal, Cumbal and Cuaspud in the Nariño Department (Colombia). Materials and Methods: As part of the research "Improving potato production as a food security contribution of native communities in Nariño, Colombia", aged October and December in 2013, an initial diagnosis of the food security situation in farmer families was conducted, through direct visits to the households selected in the sample, where in the household members, after standardization of field team, the anthropometric measures of weight and height were taken, using electronic scales, infantometers and stadiometers. Conclusions: A significant stunting was reported in children up to 7 years. In children aged 0 - 5 years group and children aged 5 - 17 years group, the results exceed by more than double the department data (according to National Survey of the Nutritional Situation in Colombia ENSIN 2010), and almost three times the national data. The underweight was generally low in all the analysed groups, although concerned the significant overweight percentage. In children aged 0 - 5 years group, the overweight data (above 2 SD) exceeded twice the department and national data according to the ENSIN 2010, although in the children aged 5 - 17 years group, the overweight and obesity was lower than the departmental and national data. In adults, the results reported that one of each two people in the range of 18 to 64 years in the five municipalities had excess weight (overweight or obesity) which was similar data respected to the department and national data. Additionally, it was noted that $18 \%$ of households were found among adults with overweight, and simultaneously, children with stunting, this situation has been called double burden of malnutri- 
tion and this is an aspect that must be analysed to identify intervention strategies.

Keywords

Nutritional Situation, Anthropometry, Stunting, Excess Weight, Nariño's Rural Families

\section{Introduction}

This project is developed as part of the research "Improving potato production as a food security contribution of native communities in Nariño, Colombia", conducted by the National University of Colombia and Mc Gill University of Canada with economical support from the International Development Research Centre (IDRC) and the Department of Foreign Affairs, Trade and Development (DFATD) of the Canadian Government, through the Canadian International Food security Research Fund (CIFSRF). It was developed in the municipalities of Pasto, Túquerres, Guachucal, Cumbal and Cuaspud in the Nariño Department (Colombia), which included in one of its initial phases, the characterization of different food security aspects in the rural households, characterization of food habits, food intake, anthropometric assessment and others. The nutritional situation is the result of the interaction aged several variables which determine the food intake and health situation, with special emphasis on infectious diseases and the different interactions aged these two factors and some unpredictable factors. Although there are several direct indicators to measure the individual nutritional situation, the alterations in growth and body dimensions, express in a systematic and reliable way, the magnitude and direction of the nutritional disorder. So, the identification of this condition is focused on nutritional anthropometry, supported in the usual anthropometric techniques.

The Nariño Department was selected to the project for combine different characteristics which included some related with agricultural production. Thereby $32.4 \%$ of the department's economy is based on agriculture, $84 \%$ of agricultural production corresponds to potato cultivation, being the third potato producer department in the country and also, the potato is part of the pattern consumption of Nariño's families. Also for the several food and nutrition problems, including the high stunting rates in children aged 0 - 5 years group (16\%) and anemia prevalence in children aged 6 - 59 months (39.4\%), according to the National Survey of the Nutritional Situation in Colombia, ENSIN 2010 [1]. With concern it notes that the overweight prevalence increases especially in schoolchildren, adolescents and adults, which constitutes a major risk for chronic diseases. This phenomenon of the undernourishment presence and simultaneously excess weight in another member of the family, especially in the mother, is what is called "double burden of malnutrition".

\section{Materials and Methods}

The data collection in field for household's characterization began on October in 2013 and ended on December of the same year. Initially was completed a questionnaire in each household that included socioeconomic information, land and territory characteristics and food consumption. For the nutritional status assessment, the weight and height were taken using electronic scales (Seca 874 electronic scale), stadiometers and infantometer (portable stadiometer Seca 217 and Seca 416 Mobile Infantometer), in the population of children aged 0 - 17 and adults aged 18 - 64 years of rural households that were selected in the households sample from the 5 municipalities of Nariño (See Map 1).

The information capture was made directly in the home. For the selection of individuals within households, a protocol was constructed to obtain representative information of children aged 0 - 17 years, pregnant women and adults aged 18 - 64 years. The total number of pregnant women that were found was included in the sample. In the first two groups all persons in the household sample of these groups were selected, while for adults aged 18 64 years, only was taken one per household. For taking the proper measures, a protocol was developed and the field team was standardized on the techniques for taking the weight and height considering the age group. Each measurement was repeated twice and in some cases up to 3 times if it was necessary according to the protocol. Finishing the assessment, was indicated to the mother or caregiver, the general measurement results and was valued the other people not included in the selection if they requested it.

For the interpretation of nutritional situation by anthropometry, the following parameters were taken into account: 


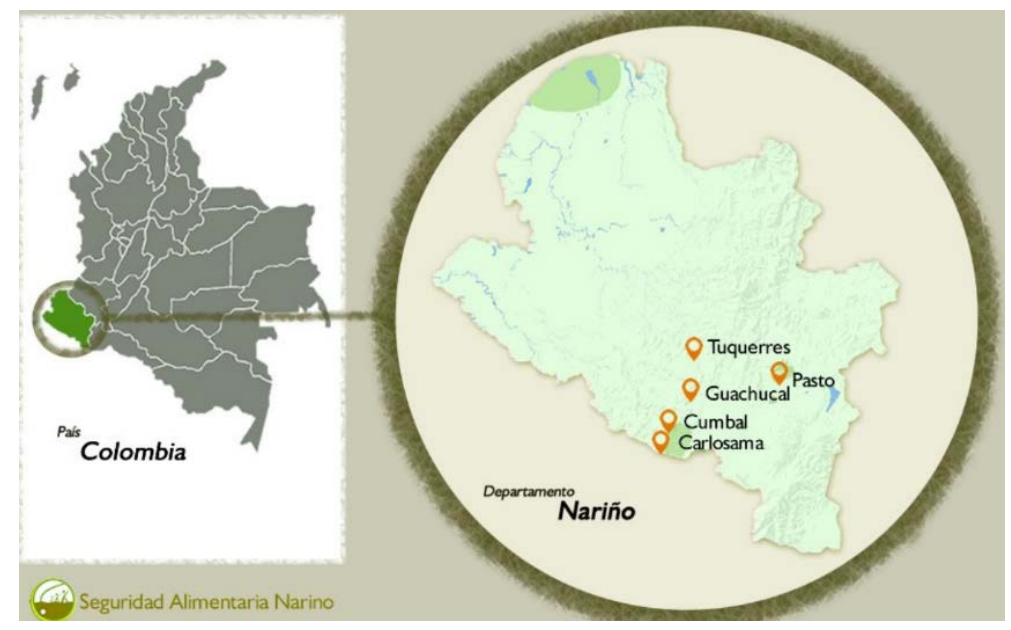

Map 1. Geographic Location of the five participating municipalities in the Project [2]. "Image: Monica Contreras: http://paradigmaweb.com/”.

In children aged 0 - 7 years, the z score indicators was calculated for each age group defined according with the Resolution 2121 of 2010, which were adopted by Colombia for the new patterns of growth and development of the World Health Organization-WHO 2006-2007 [3]. Analyzes were performed by subgroups of children aged 0 - 5 years, and 5 - 17 years using Anthro and Anthro Plus software. The used cutoff for the deficit on height and weight in both cases was $<-2$ SD, and for excess weight (overweight and obesity), 1 and 2 SD.

In people in the aged group 18-64 years, the information was analyzed using the BMI indicator, and taking the cutoff points recommended by the World Health Organization-WHO (BMI < 18.5 deficient Weight, BMI $>18.5$ and $<25$ Normal weight, BMI $>25$ and $<30$ overweight, BMI $>=30$ Obesity).

\section{Results}

In rural areas of the five municipalities of Nariño, for anthropometry component, were assessed a total of 1511 persons distributed by population groups and municipalities as is reported below in the Table 1 . The most complex age group to be found in the households was children aged 0 - 5 years.

A key aspect to consider before reporting nutritional result, is the identified ethnicity in the households. The ethnicity membership is related with the self-recognition of the respondents by their customs, traditions or by their physical features. In general it is identified that there are people who considered themselves as indigenous (Figure 1), however it is noted that these families doesn't present linguistic unit or own behaviors of indigenous communities. It is noted that of the population surveyed, the municipalities with greater recognition of its inhabitants as Indigenous are Cuaspud with $86.8 \%$ and Cumbal with $98.5 \%$, which agrees with the data reported in the Census of the National Administrative Department of Statistics-DANE 2005, where is $93 \%$ and $87.2 \%$ for each municipality respectively. Moreover, there are differences with the municipality of Guachucal that in this study reported $26.2 \%$ compared with $67.5 \%$ of the Census DANE 2005 [4].

\subsection{Nutritional Situation of Children Aged 0 - 7 Years}

The first issue reported is the stunting of children up 7 years. Figure 2 reports that in the five municipalities analyzed, just over a third part of children aged 0 - 5 years group (35\%) had stunting, being the most affected municipalities Cumbal (44.1\%) and Túquerres (42.4\%). The ENSIN 2010 reported for the Nariño Department that $16 \%$ of children aged 0 - 5 years group of the whole department were affected (13.2\% in the national level), that is to say, the results for boys and girls in the rural area of this study, in the selected municipalities, exceeds twice the data obtained at the departmental level, probably because the results were taken in the rural areas where life conditions are generally more unfavourable than urban areas, and additionally in some places, for the indigenous groups presence.

By analysing the information group of children aged 5 - 17 years, the situation is similar in importance as the children aged 0 - 5 years, the firsts reports $30.8 \%$ and the seconds $35.2 \%$ of stunting in this groups, being again 


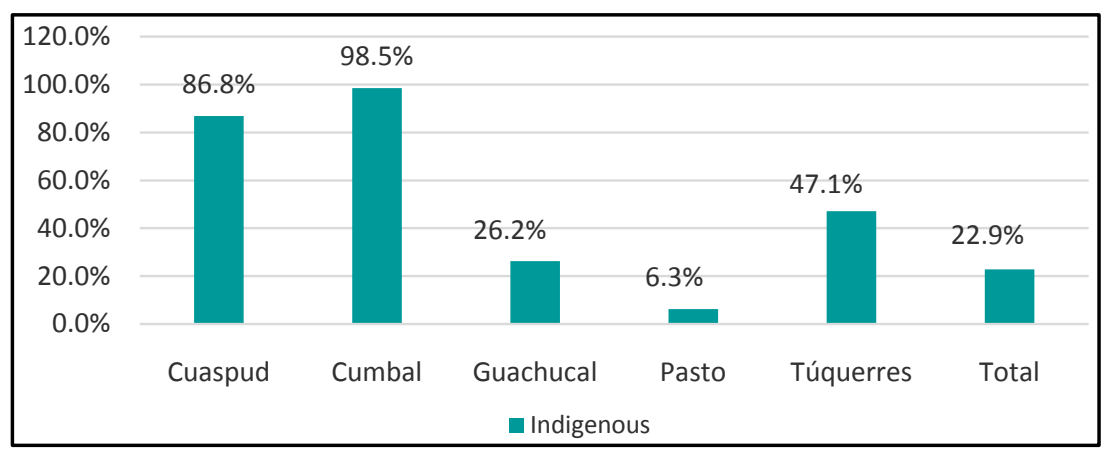

Figure 1. Percentage of indigenous households participating in the study.

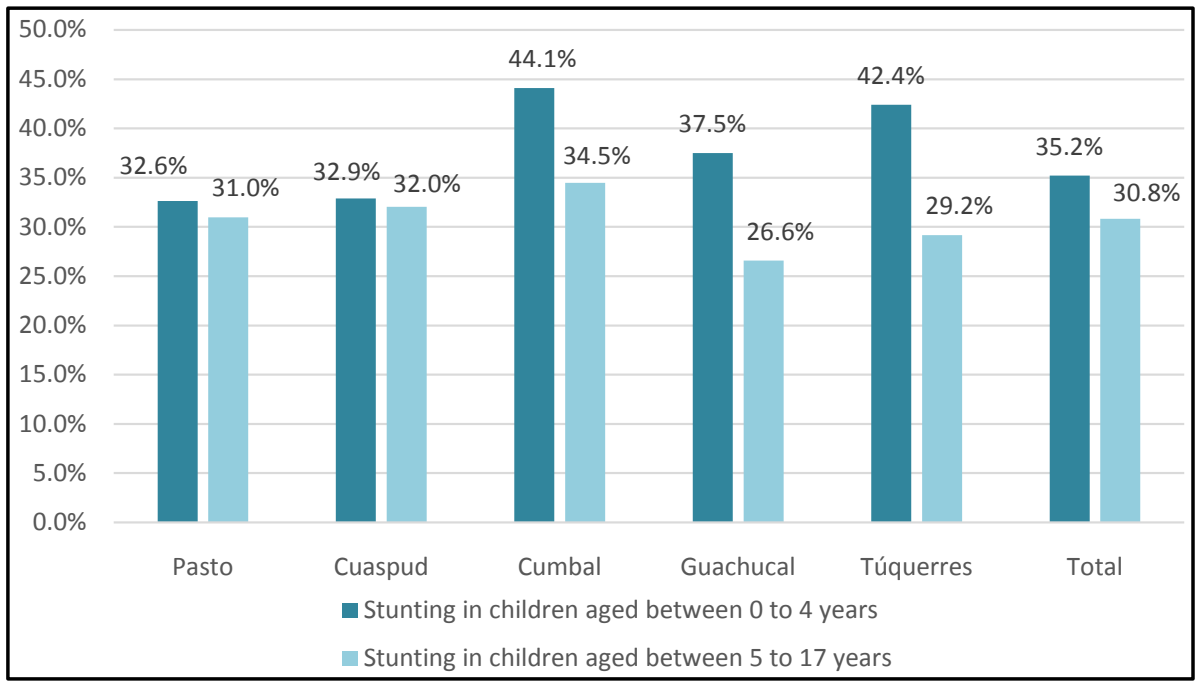

Figure 2. Stunting prevalence for children aged 0 - 4 years and 5 - 17 years of the Nariño Department. $16.9 \%$ of children aged $0-4$ years of Nariño Department have stunting or low height for age according to the ENSIN 2010. 14.1\% of children aged 5-17 years of Nariño Department have stunting or low height for age according to the ENSIN 2010.

Table 1. Distribution of the evaluated population by age groups.

\begin{tabular}{ccccccc}
\hline \multirow{2}{*}{ Age group } & \multicolumn{5}{c}{ Municipality } & Total \\
\cline { 2 - 6 } & Cuaspud & Cumbal & Guachucal & Pasto & Túquerres & \\
\hline 0 - 4 years & 62 & 58 & 27 & 68 & 52 & 267 \\
5 - 17 years & 112 & 91 & 45 & 178 & 121 & 547 \\
18 - 64 years & 155 & 115 & 72 & 193 & 162 & 697 \\
Total & 329 & 264 & 144 & 439 & 335 & 1511 \\
\hline
\end{tabular}

Age groups corresponding to the ENSIN 2010.

Cumbal (34.5\%) and Cuaspud (32.0\%) the most affected municipalities. The ENSIN 2010 reported that for this age group in the whole department (including urban and rural areas), $14.1 \%$ of them had stunting; being this data the half of these results, that only respond to the rural area. As in the children, in the ENSIN 2010 was identified that stunting data of children aged 5 - 17 years of Nariño (14.1\%) is higher than the national level (10\%), reporting the general problems of the department.

The low weight for height was reported in a low percentage in children aged 0 - 5 years group (1.2\%) similar to the national data $(0.9 \%)$. And the low weight by BMI was also lower in the group aged 5 - 17 years in the to- 
tal rural area in the five municipalities (1.6\%) versus $2.1 \%$ nationwide.

The next basic aspect to be analysed in this population group is the excess weight measured by body mass index (BMI). In children aged 0-5 years, it was observed that above 2 SD (obesity) were found $12.5 \%$ of children (Figure 3). This data is slightly more than double that the result obtained in the ENSIN 2010 for the Nariño Department (4.6\%) and for the national data (5.2\%) which may be affected in part by the high stunting in addition to all the factors related with eating habits, physical activity, etc., that may be influencing this situation.

Children aged 0 - 5 years group with the highest prevalence of excess weight (above 2 SD) are the municipalities of Pasto (13.4\%) and Cumbal (12\%).

In the children aged 5 - 17 years, obesity measured by BMI (Figure 4), was also found in 2.8\% (above 2 SD) and overweight in 10.9\% (aged 1 and 2 SD). The ENSIN 2010 reported for this population group in Nariño (added overweight and obesity) a excess weight prevalence of $17.7 \%$ (2.7\% obesity and $15.1 \%$ overweight), similar to the national data $17.5 \%$ (overweight $13.4 \%$ and obesity $4.1 \%$ ), but higher both values according with

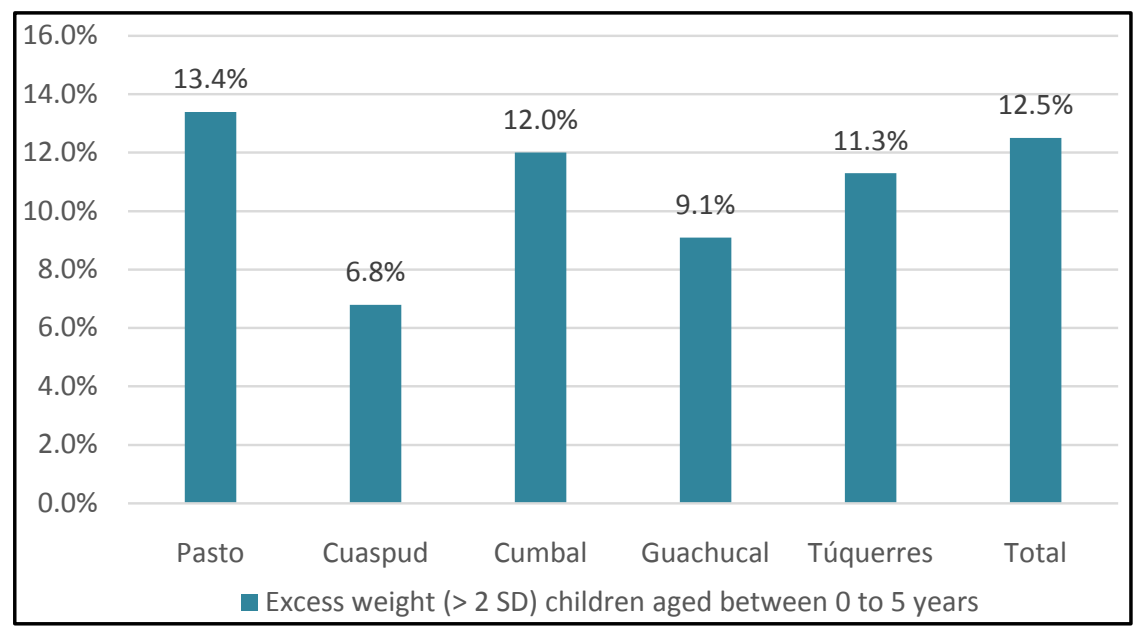

Figure 3. Excess weight prevalence in children aged 0 - 4 years of the Nariño Department. Body Mass Index Indicator (BMI). 4.6\% of children aged 0 - 4 years of the Nariño Department reports excess weight (greater than 2 SD) according to the ENSIN 2010.

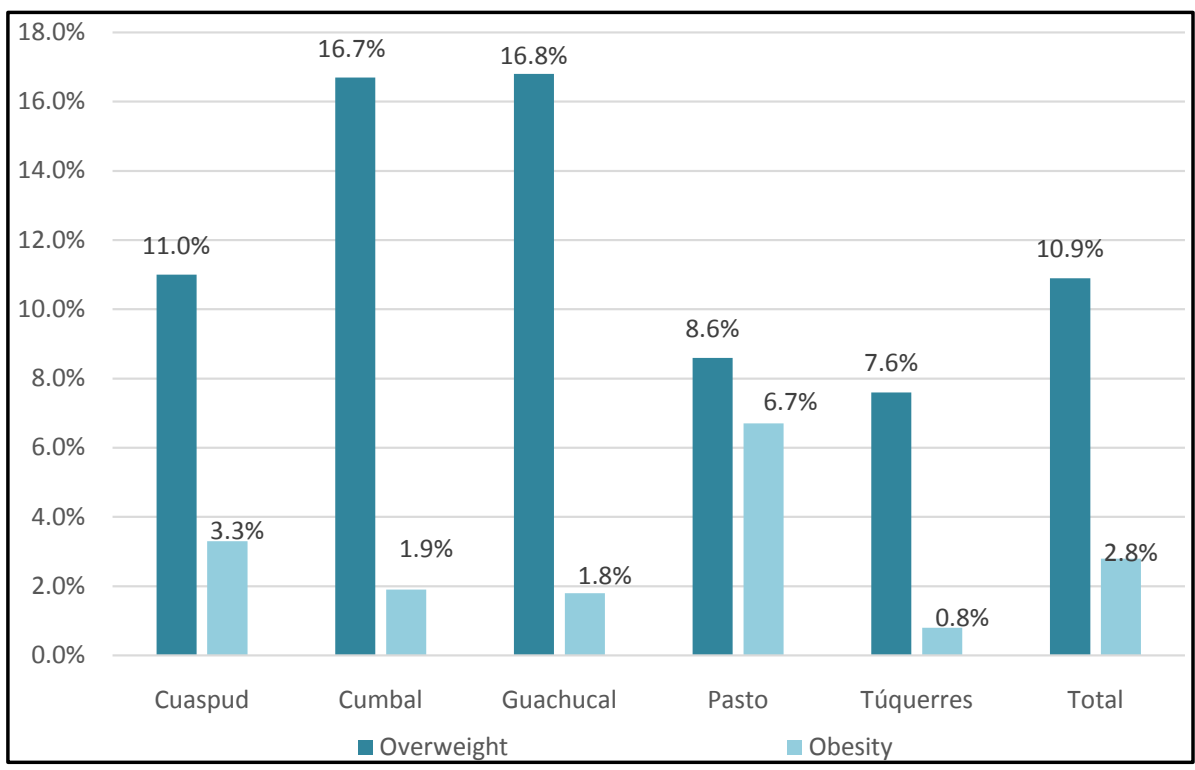

Figure 4. Excess weight prevalence in children aged 5 - 17 years of the Nariño Department. $17.7 \%$ of children aged 5 - 17 years in the department of Nariño report excess weight (overweight and obesity) according to the ENSIN 2010. 
the result obtained in this study which corresponds to the rural area (13.6\% in total).

The municipality with the highest percentage of obesity in the children aged 5 - 17 years, was Pasto in the rural area (6.7\%) and the municipalities with the highest percentage of overweight were Cumbal (16.7\%) and Guachucal (16.8\%).

\subsection{Nutritional Situation of Adults Aged 18 to 64 Years}

The results report that in the total of the five municipalities of Nariño, 38.9\% of the adults aged 18 - 64 years, have overweight measured by BMI and 12\% have obesity, which added indicates that one of each two people in this age range have some excess weight degree (Figure 5). The ENSIN 2010 reported for Nariño a similar percentage in adults (53\%; 36.6\% overweight and $16.4 \%$ obesity) as in the national data (51.2\%, overweight $34.6 \%$ and obesity $165 \%)$.

The municipalities with the adult population most affected by overweight are Guachucal (47.4\%) and Cumbal (46.4\%), and by obesity are Cumbal (15.3\%) and Cuaspud (13.0\%).

\subsection{Households with Double Burden of Malnutrition}

There are different definitions for the double burden of malnutrition. For purposes of this analysis we have taken from Barry M. Popkin, in which we define that exist the double burden of malnutrition in a household when the nutritional diagnosis of the head family woman is overweight or obesity measured by BMI, and at least some of her children present stunting for the age (chronic malnutrition) [5]. With this clarification, it was found that $18.5 \%$ of households analysed (which had anthropometric data of mother and children) had this situation (Figure 6), being the percentage even higher for municipalities of Cumbal (39.0\%) and Cuaspud (23.1\%).

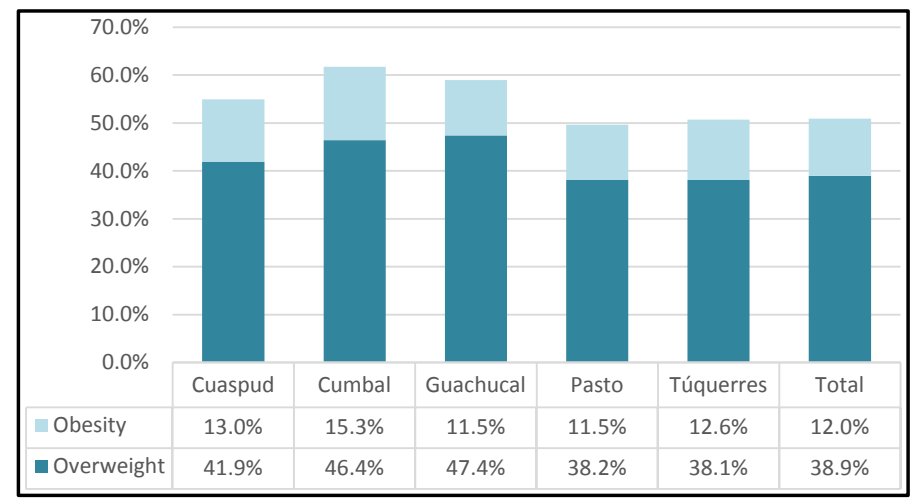

Figure 5. Excess weight prevalence in adults aged 18 - 64 years in Nariño Department. 53.0\% of adults aged 18 - 64 years in Nariño Department report excess weight (overweight and obesity) according to the ENSIN 2010.

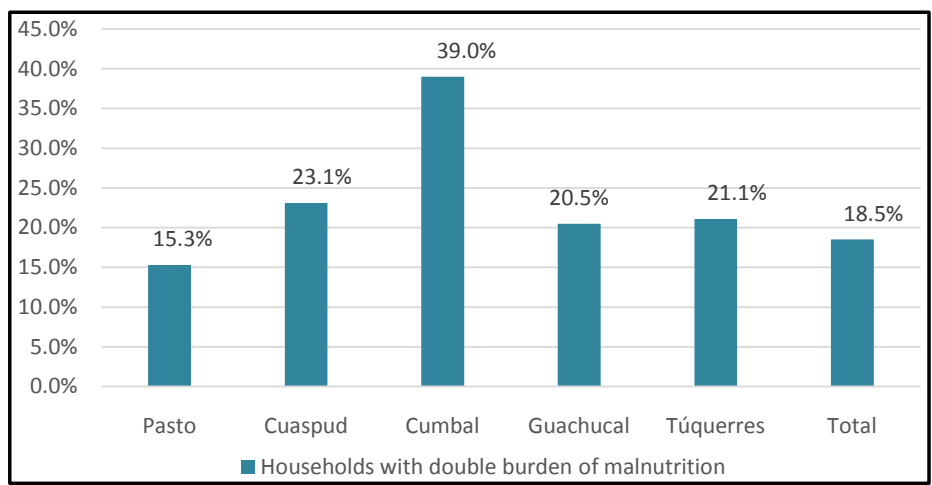

Figure 6. Households with double burden of malnutrition: Women with overweight or obesity, and children (at least one) with low height for age (chronic malnutrition or stunting). 


\section{Discussion}

One aspect in this study that should be highlighted is the robustness of the results, in reason with the sample size for the age group of children and adults which had 814 and 697 participants respectively, a proportionately higher sample that used before in the country for any study done at the municipal level.

Therefore, the results for each group examined are a reference to other similar studies, for indigenous and farmer population, especially for Andean contexts. Is notably that the studies of nutritional status in these populations are very few, which gives more significance to these results.

For the population of indigenous and farmer children aged 0 - 5 years, chronic malnutrition in this study was $35.2 \%$, similar to the situation reported in Peruvian children of the mountain range departments that reported $30,7 \%$ in 2011 [6]. These data are consistent also with the population of Bolivia, where most of their population is Andean, and reports $40.6 \%$ of chronic malnutrition [7]. This aspect reports the high rates of stunting that afflicts children, where the determinants of this situation are being shared in these contexts associated with Andean and farmer population, with a strong indigenous presence.

In this age group, the percentage of excess weight was $12.5 \%$, similar to the data that was reported in [8], the data for the mountain range population in Peru was $8.2 \%$, which is high for this age group. This indicates the presence of excess weight in contexts of poverty for populations of the same Andean contexts, both in Nariño Department as in Peru.

Another finding of this study is the high data of excess weight in the population aged 5 and 18 years who reach $13.7 \%$, however, in this age group is not high the prevalence than that was reported in [8] which exceeds the $38 \%$ in the same age group in the Peruvian Andine mountain range region.

For the adult population in the rural area of Andine regions, the information is scarce. However it seems like the excess weight behavior is high in both areas, how was demonstrated by the ENSIN 2010, where 52.5\% of adult population in the urban area presented overweight or obesity (BMI > 25) compared with $46.9 \%$ of the population in the rural area.

In this study the double burden of malnutrition was calculated, namely the percentage of households where there was at least one obese or overweight adult and simultaneously there was the presence of a child with chronic malnutrition or stunting. Particularly worrisome results reports a prevalence of $18.2 \%$, more than five percentage points above the data of double burden of malnutrition for the Nariño Department, with 13.53\%, [9], in a calculation done from the ENSIN 2010 databases, indicating that the situation of double burden of malnutrition, of the Andean households in the examined region in this study is higher than the regional and national data, meriting this contexts an urgent intervention.

\section{Conclusions}

The results clearly present that farmers and indigenous families of the Nariño's municipalities included in the study, report an important malnutrition problem, both deficit and excess which must be taken into account when working policies, plans and programs aimed to improve in general, the food security conditions of these populations, view from their different components. This study noticed a significant stunting of children up to 7 years. So, in children aged 0 - 5 years and children aged 5 - 17 years, the results exceed by more than double the results of the department (according to the ENSIN 2010) and almost three times the national data. Although the ENSIN 2010 survey was not representative for all indigenous groups of the country, it worked to report the situation of indigenous groups that was in the sample of this study, and there was identified that stunting had more than doubled of the children aged 0 - 5 years group of this population ( $29.5 \%$ vs.13.2\% in the national level) and in the group aged 5 - 17 years, almost triple of the rate of stunting ( $29 \%$ vs. $10 \%$ in the national level). Similarly the study noticed significant differences aged the results from urban areas rural according with rural areas, the latter being always the most affected. It is important to remember that the stunting or chronic malnutrition is a condition which indicates a food and nutrition insecurity problem in long term, and has a cumulative character over the time, especially when social, economic, health and environmental conditions, have been deficient for a prolonged time period.

The underweight is generally low in all the different groups analysed, but it notes with concern that excess weight is a growing every time, situation that as the deficit, brings multiple problems especially related with the chronic diseases. In children aged 0 - 5 years group the overweight data (above 2 SD) exceeded twice the department and national data according to the ENSIN 2010, although in the group aged 5 - 17 years, overweight 
and obesity was lower than departmental and national information. In adults, the results reported that one of each two people in the range of 18 to 64 years in the five municipalities had excess weight (overweight or obesity), similar data with the departmental and national level. The presence in the households of adults with overweight and children with stunting is what has been called double burden of malnutrition, and is an aspect which must be analysed to identify intervention strategies. It is necessary to revise in this population, the structural aspects that may be affecting their stunting situation, but more important yet is their lifestyle, habits and dietary costumes, physical activity performed, which may influence the presented overweight.

\section{References}

[1] Social Protection Ministery, Colombian Family Welfare Institute, Health National Institutes (2010) National Survey of the Nutritional Situation in Colombia. ENSIN 2010.

[2] Contreras, M. (2014) Map of Municipalities Participating in the Study "Improving Potato Production as a Food Security Contribution of Native Communities in Nariño, Colombia”.

[3] WHO (2010) Whereby Were Taken the Growth Patterns Published by World Health Organization for Children and Adolescents Aged 0 to18 Years of Age.

[4] DANE (2005) National Population Census.

[5] Popkin, P. (1998) The Nutrition Transition and Its Health Implications in Low-Income Counttries. 5-21.

[6] Sánchez Abanto, J. (2012) Evolution of Chronic Malnutrition in Children under Five in Peru. Revista Peruana de Medicina Experimental y Salud Publica, 29, 40.

[7] Institute-Programme The World Food (2006) Survey Results of the Food and Nutrition Security in the Most Vulnerable Municipalities in Bolivia. La Paz.

[8] Álvarez-Dongo, D., Sánchez-Abanto, J. and Gómez-Guizado, G. (2012) Overweight and Obesity: Prevalence and Determinants of Overweight in the Peruvian Population. Revista Peruana de Medicina Experimental y Salud Publica, 29, 303-313.

[9] Ministry of Health of Colombia (2014) Monitoring Food and Nutrition Security in Colombia (OSAN), Approach to the Determinants of Dual Nutritional Burden in Colombia. 
Scientific Research Publishing (SCIRP) is one of the largest Open Access journal publishers. It is currently publishing more than 200 open access, online, peer-reviewed journals covering a wide range of academic disciplines. SCIRP serves the worldwide academic communities and contributes to the progress and application of science with its publication.

Other selected journals from SCIRP are listed as below. Submit your manuscript to us via either submit@scirp.org or Online Submission Portal.
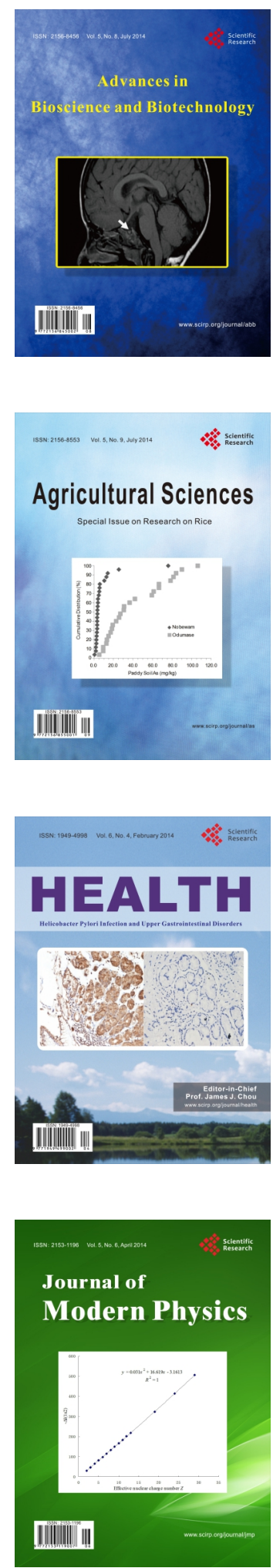
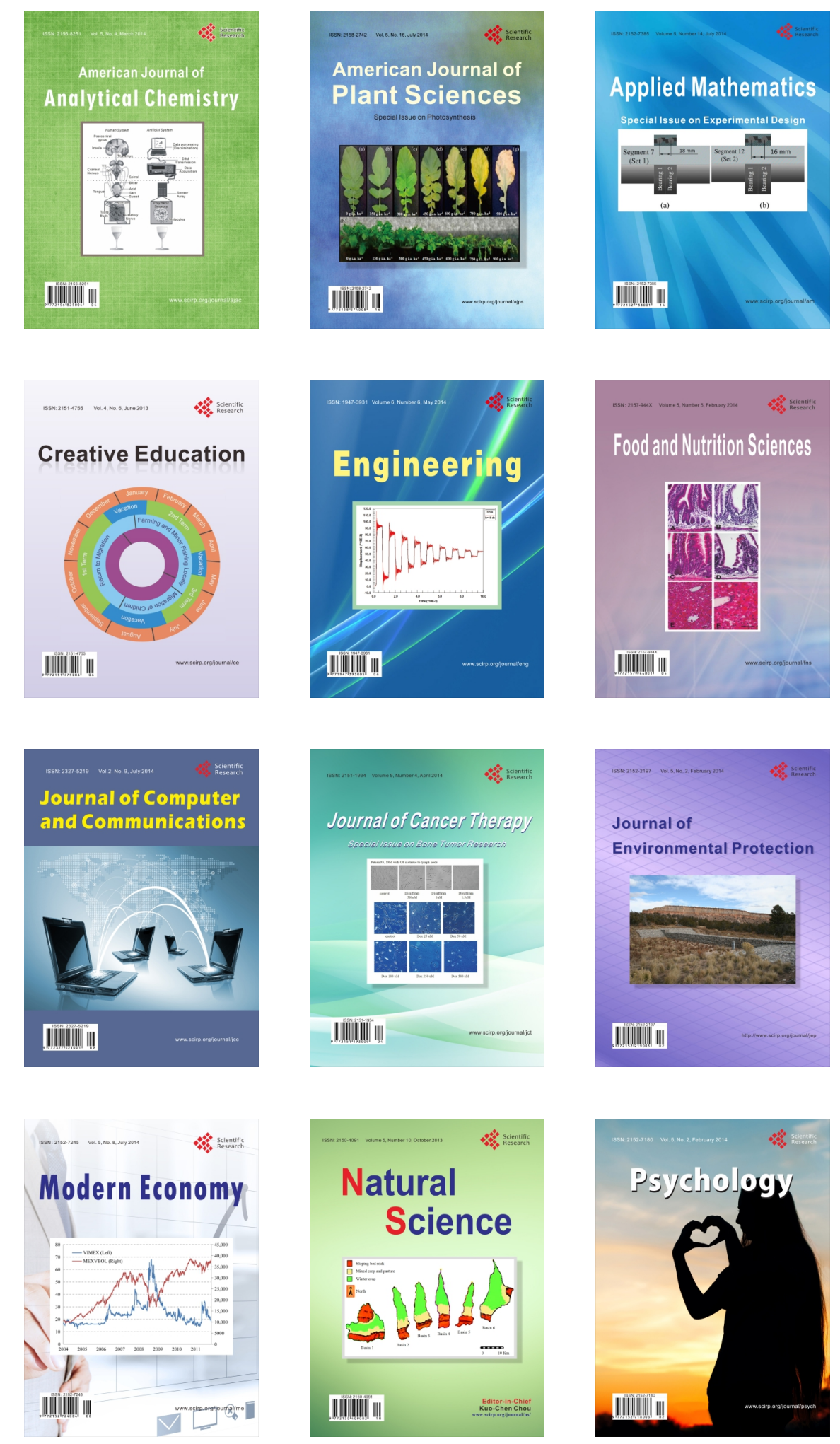